\title{
Mortality Rate and Predictors among Patients with COVID-19 Related Acute Respiratory Failure Requiring Mechanical Ventilation: A Retrospective Single Centre Study
}

\author{
Christophe de Terwangne ${ }^{1 *}$, Antonio Sorgente ${ }^{2}$, Redente Tortora ${ }^{3}$, Diana Cheung ${ }^{3}$, \\ Frederic Duprez ${ }^{3}$, Sammy Place ${ }^{1}$, Jerome R. Lechien ${ }^{4}$, Lucio Capulzini², Michael De \\ Cubber $^{2}$, Sven Saussez ${ }^{4}$, Fabio Silvio Taccone ${ }^{5}$, Shahram Mashayekhi ${ }^{3}$ \\ 1 Department of Internal Medicine, Centre Hospitalier EpiCURA Hornu, Hornu, Belgium \\ 2 Department of Cardiology, Centre Hospitalier EpiCURA, Hornu, Belgium \\ 3 Department of Intensive Care, Centre Hospitalier EpiCURA, Hornu, Belgium \\ 4 Department of Human Anatomy and Experimental Oncology, University of Mons, Mons, Belgium \\ 5 Department of Intensive Care, Hopital Erasme, Université Libre de Bruxelles, Brussels, Belgium
}

\begin{abstract}
Aim: The objective of the study was to assess mortality rates in COVID-19 patients suffering from acute respiratory distress syndrome (ARDS) who also requiring mechanical ventilation. The predictors of mortality in this cohort were analysed, and the clinical characteristics recorded. Material and method: A single centre retrospective study was conducted on all COVID-19 patients admitted to the intensive care unit of the Epicura Hospital Center, Province of Hainaut, Belgium, between March 1st and April 30th 2020. Results: Forty-nine patients were included in the study of which thirty-four were male, and fifteen were female. The mean (SD) age was 68.8 (10.6) and 69.5 (12.6) for males and females, respectively. The median time to death after the onset of symptoms was eighteen days. The median time to death, after hospital admission was nine days. By the end of the thirty days follow-up, twenty-seven patients (55\%) had died, and twenty-two (45\%) had survived. Non-survivors, as compared to those who survived, were similar in gender, prescribed medications, COVID-19 symptoms, with similar laboratory test results. They were significantly older $(p=0.007)$, with a higher co-morbidity burden $(p=0.026)$ and underwent significantly less tracheostomy $(p<0.001)$. In multivariable logistic regression analysis, no parameter significantly predicted mortality. Conclusions: This study reported a mortality rate of 55\% in critically ill COVID-19 patients with ARDS who also required mechanical ventilation. The results corroborate previous findings that older and more comorbid patients represent the population at most risk of a poor outcome in this setting.
\end{abstract}

Keywords: mortality, critically ill, mechanical ventilation, coronavirus, SARS-COV-2

Received: 5 August 2020 / Accepted: 22 November 2020

\section{INTRODUCTION}

Since the first report in December 2019 of the cluster of pneumonia of unknown origin in Wuhan, China, in January 2020, a novel pathogen was identified as the Severe Acute Respiratory Syndrome Coronavirus-2 (SARS-CoV-2). The continually increasing number of cases outside China alerted the World Health Organization, and a pandemic outbreak was declared on March $11^{\text {th }} 2020[1]$.

The disease renamed COVID-19, can remain asymptomatic, but in many cases, it can cause severe hypox- emia, which may rapidly lead to an acute respiratory distress syndrome (ARDS) and require the admission to the Intensive Care Unit (ICU) [2]. Since the onset of the pandemic, many critically ill patients have been treated across Europe, including Belgium [3, 4]. The Borinage region, located in the province of Hainaut in Belgium, has been significantly affected during the March to May Belgian outbreak of SARS-COV-2 [5].

The regional hospital, a primary care centre, admitted around 400 COVID-19 patients with concomitant respiratory failure. Fifty-two of them were admitted to the ICU and placed on mechanical ventilation. 
Recent studies have shown that the use of mechanical ventilation among critically ill COVID-19 patients admitted to an ICU ranged between $29 \%$ and $90 \%$ [6] and mortality rates varied from $16 \%$ to $97 \%$ [7], although in most of the reported studies patients were still hospitalised at the time of the report. Furthermore, ICU admission criteria vary widely among different caring centres or institutions, making data comparison and interpretation difficult. Hence, little is known about the actual mortality rate patients with COVID-19 related ARDS.

This study aimed to investigate mortality rates in patients with COVID-19 related acute respiratory failure requiring mechanical ventilation.

\section{MATERIAL AND METHODS}

All critically ill COVID-19 patients admitted to the ICU of the Epicura Hospital Center, Province of Hainaut, Belgium, between March $1^{\text {st }}$ and April 30th 2020, were assessed for inclusion in this single centre retrospective study. The local Ethics Committee of the hospital approved the study protocol (P2020/024) and waived the need for obtaining patients' written informed consent.

The study was performed following the ethical standards of the 1964 Declaration of Helsinki and its later amendments.

Patients were included in the study if they were positive on a real-time polymerase chain reaction test (RTPCR) on a nasopharyngeal swab for SARS-COV-2, presented SARS-COV-2 related hypoxemic respiratory failure or ARDS following Berlin's criteria, and underwent mechanical ventilation.

Patients were excluded if they were admitted to ICU and mechanically ventilated for other reasons than COVID-19 related ARDS, or if their survival status, at the end of follow-up, was unknown.

The date of the last follow-up was June $26^{\text {th }} 2020$.

On that day, an outcome was assigned to each patient (a) deceased (b) discharged-alive.

The discharged-alive patients subsequently were contacted by telephone to evaluate their vital status, following routine hospital practice.

The reason for the initiation of mechanical ventilation was based on oxygen saturation $\left(\mathrm{SpO}_{2}\right)$ levels $<$ $90 \%$ on maximum oxygen delivery, i.e. equivalent of $\mathrm{FiO}_{2}>80 \%$ delivered via a T-piece mask or non-rebreathing mask, in the presence of the clinical signs of respiratory failure, i.e. increased respiratory rate $>35$ rpm, altered consciousness, mottling skin.
On admission, patients were ventilated using a protective mode of the volume control, using a tidal volume of $6 \mathrm{ml} / \mathrm{kg}$ (ideal body weight) and limitation of plateau pressure below $30 \mathrm{cmH}_{2} \mathrm{O}$. Afterwards, ventilation modes were adjusted according to the clinical evolution and the judgement of the senior ICU physician.

The decision and timing of tracheostomy were not standardised and was taken in a collegial fashion after simultaneous evaluation of the duration of mechanical ventilation, $\mathrm{a} \mathrm{FiO}_{2}>50 \%$, persistent radiological abnormalities and the hemodynamic stability of each patient.

Medical treatment was decided by the senior treating physician in collaboration with the infectious diseases specialist.

Medication included Plaquenil ${ }^{\circledR}$ (Sanofi, Paris, France) $400 \mathrm{mg}$ orally twice a day on the first day followed by $200 \mathrm{mg}$ twice a day until the fifth day.

Data were retrieved from the electronic medical records by three critical-care physicians who were directly involved in the care of the patients and include demographics, comorbidities, clinical characteristics, pharmacological treatment, laboratory results, results of radiologic examinations and complications. The burden of comorbidities was defined according to the Charlson comorbidity index score [10], developed to predict ten-year survival in adults. The cause of death, i.e. respiratory, hemodynamic, neurological or withdrawal of life-sustaining therapies, was adjudicated by all the authors after an in-depth review of each case.

ARDS and ARDS severity classification was defined according to Berlin's criteria [8] and severity of COVID-19 according to the World Health Organization criteria [11].

Descriptive statistics were computed for all study variables. Discrete variables were expressed as percentages and continuous variables as mean (SD) or median [25th-75th percentiles]. Difference between survivors and non-survivors were assessed using the chi-square test or Fisher's exact test for categorical variables, and Student's t-test or Mann-Whitney U-test for continuous variables. Analysis of mortality over the follow-up time was assessed by the Kaplan Meyer curve. Multivariable logistic regression analysis, with mortality as the dependent variable, was performed for all patients. Collinearity between variables, i.e. a variance inflation factor greater than five, was excluded before modelling; only variables associated with mortality in the univariate analysis were included in the multivariate model. The odds ratio, with $95 \%$ confidence intervals, was computed. 
The significance level was set at $\boldsymbol{\alpha}=0.05$, and a p-value below 0.05 was considered statistically significant.

Statistical analyses were performed using the SPSS 20.0 (SPSS, Inc., Chicago, Illinois, US).

\section{RESULTS}

From March $1^{\text {st }} 2020$ to April $30^{\text {th }} 2020$, a total number of 52 patients were admitted to the ICU. Three patients were excluded because of unknown outcomes at the end of the follow-up; therefore, the final analyses were conducted on forty-nine patients.

The study included thirty-four male and fifteen female patients. Mean (SD) age was 68.8 (10.6) and 69.5 (12.6) for males and females, respectively.

All patients had at least one comorbidity. Most common self-reported COVID-19 symptoms were fever (76\%), dyspnoea (71\%) and cough (69\%). Median time from the first self reported symptoms to the commencement of mechanical ventilation and from hospital admission to the commencement of mechanical ventilation were 7.5 (5.8) and 2 (2.7) days, respectively.

Thirty-three patients suffered from severe ARDS, and twelve suffered from moderate ARDS and four from mild ARDS.

There was no statistical difference in overall mortality among these groups $(19 / 33,57 \%$ vs $7 / 12,58 \%$ vs $1 / 4,25 \%$; respectively. ( $\mathrm{p}=0,450$; Chi-square test with an adjusted $\mathrm{p}$-value for column proportion comparison according to Bonferroni method.)
At the end of follow-up, twenty-seven patients (55\%) had died, and twenty-two were alive (45\%). The median time to death after the onset of symptoms was eighteen days. The median time to death after hospital admission was nine days (Figure 1). The main reason of death was respiratory failure in nineteen patients (70\%) of which eight required inotropic support at the time of death, circulatory failure in three patients (11\%), cardiac arrest in three patients (11\%) and was undetermined in two patients $(8 \%)$.

The median duration of mechanical ventilation was 16 (16.3) and 8 (12.5) days in survivors and non-survivors, respectively.

Twenty-one patients out of forty-nine suffered from ventilation acquired pneumonia, which was less frequent in non-survivors than survivors $(\mathrm{p}=0.002$; Chisquare test).

Table 1 shows that non-survivors compared to survivors, had similar demographics, chronic disease medication, COVID-19 symptoms or laboratory- test abnormalities on admission, but were significantly older ( $p=0.007$; Mann-Whitney U Test), had more comorbidities, according to the Charlson index, $(\mathrm{p}=0.026$; Mann-Whitney U Test,) and were less likely to receive tracheostomy $(\mathrm{p}<0.001$; Chi-square test $)$.

Furthermore, the length of stay in the ICU and the duration of mechanical ventilation were significantly longer in survivors $(\mathrm{p}<0.001$ and $\mathrm{p}=0.006$, respectively; Mann-Whitney U Test). In multivariable logistic

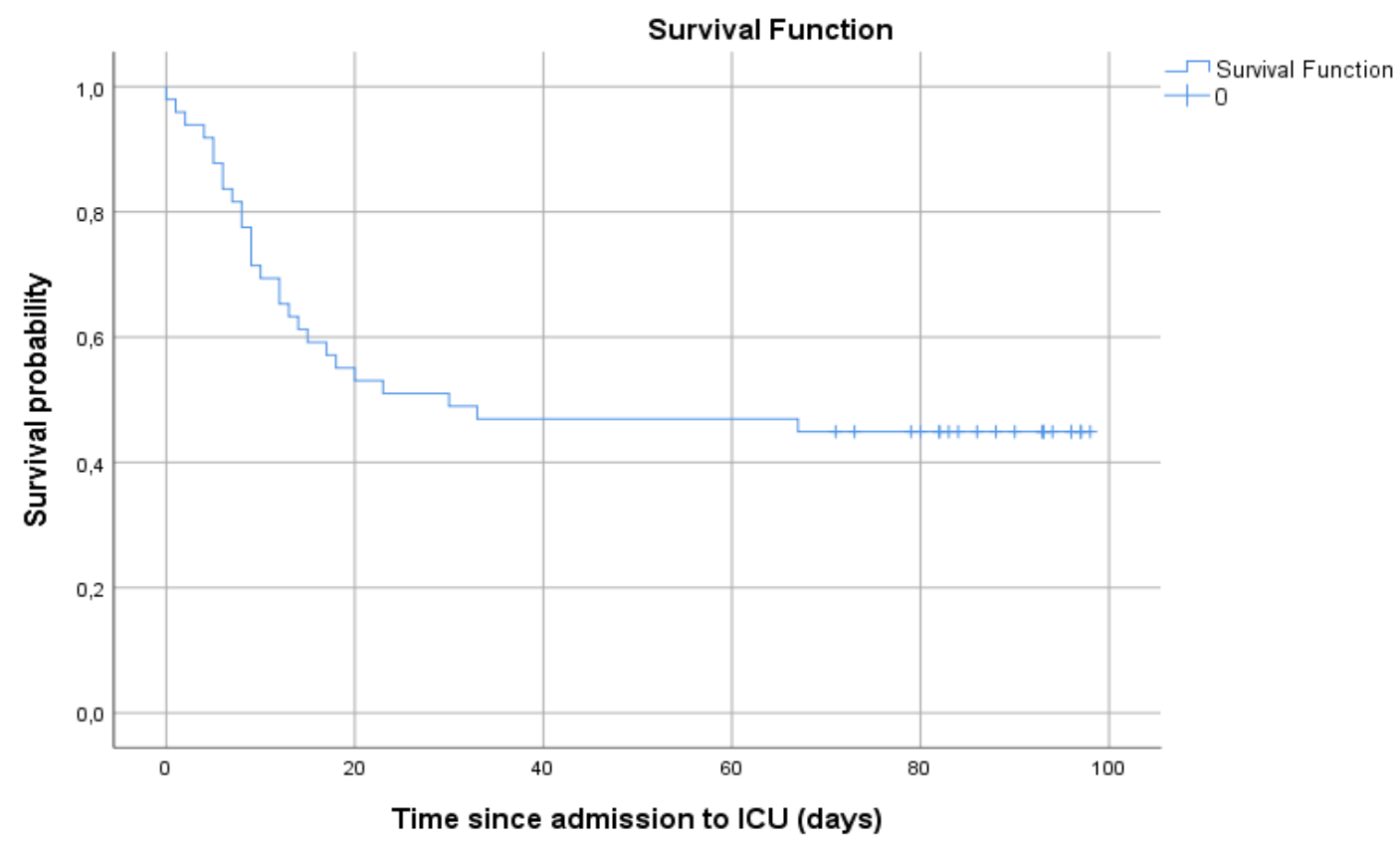

Fig. 1. Kaplan-Meier survival curve after admission to ICU. 
Table 1. Baseline and clinical characteristics of COVID-19 patients, according to the overall outcome.

\begin{tabular}{|c|c|c|c|c|}
\hline & All $(n=49)$ & Survivors ( $n=22$ ) & Non-Survivors ( $n=27$ ) & p-value \\
\hline \multicolumn{5}{|l|}{ Demographic data } \\
\hline Age & $71(61,5-77)$ & $66,50(54,3-71,3)$ & $73(65-80)$ & 0.007 \\
\hline Age $>71$ years & $22(45 \%)$ & $5(23 \%)$ & $17(63 \%)$ & 0.009 \\
\hline Female Gender & $15(31 \%)$ & $7(32 \%)$ & $8(30 \%)$ & 1.00 \\
\hline \multicolumn{5}{|l|}{ Time spans } \\
\hline $\begin{array}{l}\text { Time from symptoms to mechanical ventilation } \\
\text { (days) }\end{array}$ & $7.5(5-12)$ & $8.5(6-11.75)$ & $7(3.5-11.5)$ & 0.226 \\
\hline $\begin{array}{l}\text { Time from hospital admission to mechanical } \\
\text { ventilation (days) }\end{array}$ & $2(1-5)$ & $2.5(0-4.75)$ & $2(1-5)$ & 0.573 \\
\hline \multicolumn{5}{|l|}{ Chronic Medications } \\
\hline Oral antidiabetic & $10(20 \%)$ & $4(18 \%)$ & $6(22 \%)$ & 1.000 \\
\hline Insulin & $6(12 \%)$ & $3(14 \%)$ & $3(11 \%)$ & 1.000 \\
\hline Antiplatelet therapy & $15(31 \%)$ & $11(41 \%)$ & $4(18 \%)$ & 0.123 \\
\hline Anticoagulation therapy & $6(12 \%)$ & $4(15 \%)$ & $2(9 \%)$ & 0.678 \\
\hline RAAS inhibitor & $16(33 \%)$ & $5(23 \%)$ & $11(41 \%)$ & 0.229 \\
\hline \multicolumn{5}{|l|}{ Comorbidities } \\
\hline History or active smoking & $19(39 \%)$ & $7(32 \%)$ & $12(44 \%)$ & \\
\hline COPD & $3(6 \%)$ & $2(9 \%)$ & $1(4 \%)$ & 1.000 \\
\hline Sleep Apnoea & $10(20 \%)$ & $5(23 \%)$ & $5(19 \%)$ & 0.726 \\
\hline Asthma & $4(8 \%)$ & $2(9 \%)$ & $2(7 \%)$ & 1.000 \\
\hline Diabetes & $20(41 \%)$ & $10(45 \%)$ & $10(37 \%)$ & 0.574 \\
\hline Dyslipidemia & $25(51 \%)$ & $12(55 \%)$ & $13(48 \%)$ & 0.573 \\
\hline Cardiovascular-cerebrovascular disease & $15(31 \%)$ & $10(37 \%)$ & $5(23 \%)$ & 0.358 \\
\hline Chronic Ischemic heart disease & $7(14 \%)$ & $2(9 \%)$ & $5(19 \%)$ & 0.436 \\
\hline Hypertension & $32(65 \%)$ & $13(59 \%)$ & $19(70 \%)$ & 0.548 \\
\hline History of stroke & $5(10 \%)$ & 2 (9\%) & $3(11 \%)$ & 1.000 \\
\hline Peripheral Arterial disease & $7(14 \%)$ & $2(9 \%)$ & $5(19 \%)$ & 0.436 \\
\hline Hepatopathy or cirrhosis & $2(4 \%)$ & $1(5 \%)$ & $1(4 \%)$ & \\
\hline History of cured cancer & $6(12 \%)$ & $3(14 \%)$ & $3(11 \%)$ & 1.000 \\
\hline Actively treated cancer & $1(2 \%)$ & $0(0 \%)$ & $1(4 \%)$ & 1.000 \\
\hline Chronic kidney disease & $8(16 \%)$ & $3(14 \%)$ & $5(19 \%)$ & 0.715 \\
\hline Immunosuppression & $6(12 \%)$ & 2 (9\%) & $4(15 \%)$ & 0.678 \\
\hline Charlson score & $4(2-6)$ & $2.5(1-4.75)$ & $5(3-6)$ & 0.026 \\
\hline Charlson score $>2$ & $34(69 \%)$ & $11(50 \%)$ & $23(85 \%)$ & 0.012 \\
\hline \multicolumn{5}{|l|}{ Symptoms at admission } \\
\hline Fever & $37(76 \%)$ & $18(82 \%)$ & $19(70 \%)$ & 0.507 \\
\hline Cough & $34(69 \%)$ & $17(77 \%)$ & $17(63 \%)$ & 0.358 \\
\hline Dyspnoea & $35(71 \%)$ & $14(64 \%)$ & $21(78 \%)$ & 0.348 \\
\hline Chest pain & $5(10 \%)$ & 2 (9\%) & $3(11 \%)$ & 1 \\
\hline Dysgeusia/Anosmia & $5(10 \%)$ & $3(14 \%)$ & $2(7 \%)$ & 0.646 \\
\hline Myalgia & $18(37 \%)$ & $9(41 \%)$ & $9(33 \%)$ & 0.767 \\
\hline Headache & $11(22 \%)$ & $7(32 \%)$ & $4(15 \%)$ & 0.185 \\
\hline Nausea & $9(18 \%)$ & $5(23 \%)$ & $4(15 \%)$ & 0.713 \\
\hline Vomiting & $6(12 \%)$ & $3(14 \%)$ & $3(11 \%)$ & 0.314 \\
\hline Abdominal pain & $4(8 \%)$ & $2(9 \%)$ & $2(7 \%)$ & 1 \\
\hline Diarrhoea & $11(22 \%)$ & $6(27 \%)$ & $5(19 \%)$ & 0.510 \\
\hline \multicolumn{5}{|l|}{ Analysis of blood gases at admission } \\
\hline Litres of $\mathrm{O}_{2}$ administered at admission in ICU & $10(6-15)$ & $8(4-15)$ & $11(7-15)$ & 0.071 \\
\hline $\mathrm{pH}$ & $7.45(7.36-7.48)$ & $7.47(7.39-7.51)$ & $7.42(7.34-7.47)$ & 0.075 \\
\hline
\end{tabular}




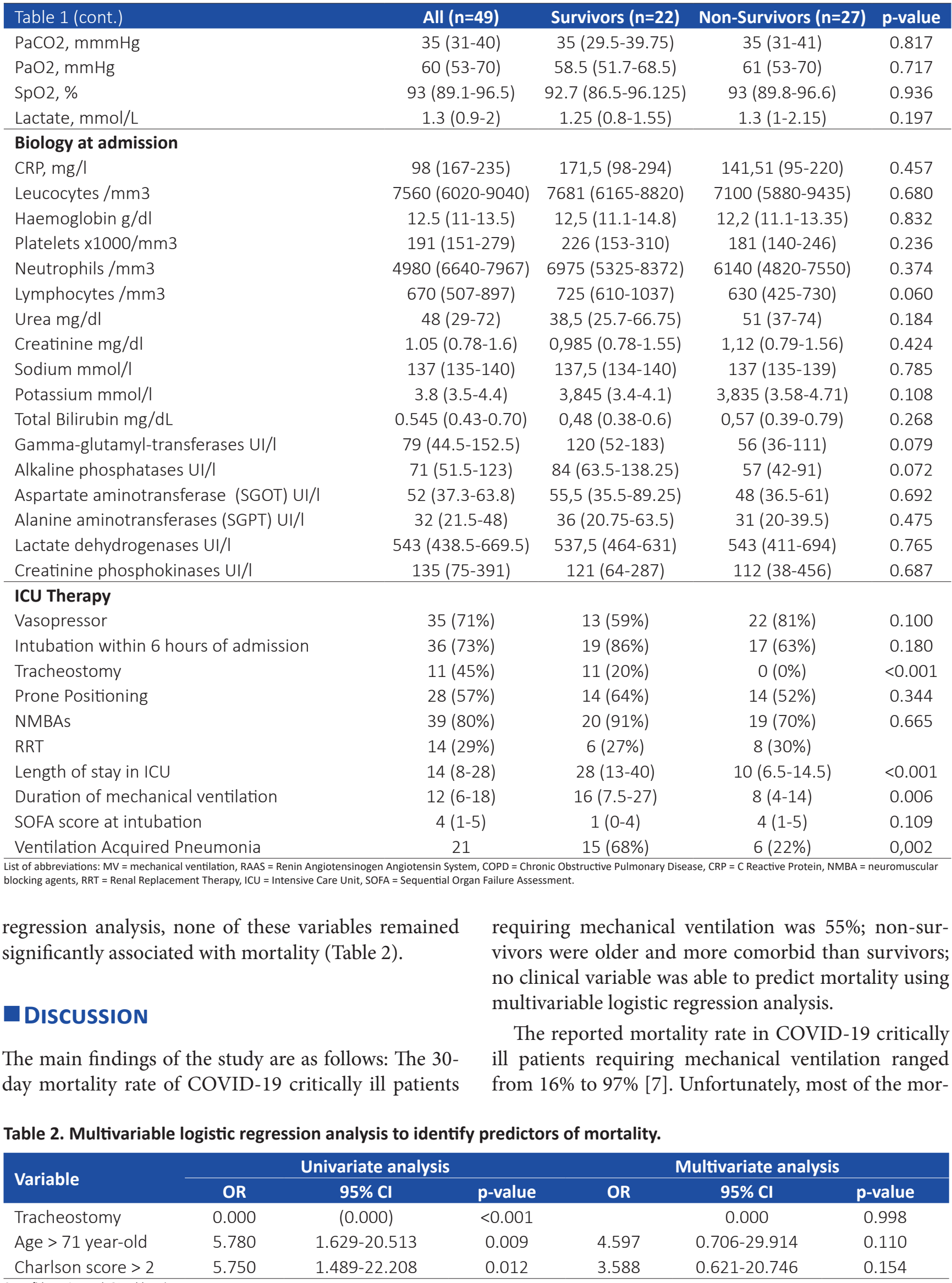


tality rates were calculated on case-series which lack data on the whole cohort.

Of the fifteen studies reviewed by Quah et al. (2020) regarding ICU and mechanical ventilation associated mortality in the settings of COVID-19 related ARDS, all include patients still in ICU at the time of data publication; in some of these reports up to $82 \%$ of patients were still hospitalised at the time of reporting [7]. This finding may lead to an inappropriate estimation of mortality rates $[6,7]$. In the current retrospective analysis, the results showed a mortality rate of $55 \%$ for ARDS associated with COVID-19 pneumonia requiring mechanical ventilation, which is significantly lower than those reported in early Chinese studies [12-14]. This difference may be explained by the implementation of many therapeutic interventions, which were unknown at the time when the first Chinese patients were treated.

Additional outcome analyses are necessary to understand how therapeutic interventions may have changed the courses and clinical outcomes of this patient population.

Because of the high risk of aerosolisation of viral particles, the lack of evidence on the efficacy of noninvasive ventilation at the time of the first epidemiological peak and general in-house recommendations at that time, devices such as high-flow nasal oxygen delivery or continuous positive airways pressure ventilation were not used $[8,9]$. Moreover, since there was a shortage in ICU staff and beds at that time, all patients with a $\mathrm{SpO}_{2}>90 \%$, regardless of the oxygen supply, but without clinical signs of respiratory failure, were not admitted to the ICU. Furthermore, in the present study, intubation was postposed as much as possible, predicated on the high mortality reported in earlier publications, the potential risks of ventilation-induced lung injury and ventilator-associated pneumonia.

It is important to recognise that, despite an effort to delay intubation, the median time between hospital admission and initiation of mechanical ventilation was only two days. Every effort must be made to identify critically ill-hospitalised patients with Covid-19 related pneumonia as these patients can destabilise quickly.

The current study data also demonstrated a skewed distribution of the mortality, with $88 \%$ of deaths occurring in the first twenty days. It seems that once patients do survive acute respiratory failure and an initial acute cytokine storm [15], as well as immediate complications such as thromboembolic complications [16] or acute cardiac injury [17], their clinical status progressively improves by the third week. This could also explain why patients developing ventilator-associated pneumonia have a lower mortality rate than others. Indeed, as very sick patients frequently die during the first days after admission, there has been little time for late infectious complications to develop.

All eleven patients in the study, who underwent a tracheostomy, survived. This could just reflect the decision to perform a tracheostomy in those survivors after a prolonged ICU-stay to facilitate mechanical ventilation weaning and rehabilitation.

It has noted that non-survivors were older and more comorbid than survivors. The Charlson comorbidity index score was significantly higher in non-survivors, suggesting a role for pre-existing comorbidity- burden on patients' outcome.

Age greater than 71-year-old, a Charlson score greater than 2 and tracheostomy were the only variables which showed a significance in the univariate analysis. However, when combined in the multivariate model, no clinical predictor of mortality could be identified. These findings are not surprising because of the small number of patients included in this retrospective analysis. A more numerous population would have helped in the identification of possible predictors of poor outcome.

A wide variety of predictive factors of mortality of critically ill COVID-19 patients have already been published. Variables such as age, thrombocytopenia, D-dimer, lactates, potassium, ARDS severity, creatinine level, acute kidney injury, and comorbidity factors such as ischemic heart disease $[18,19]$, have been demonstrated to be relevant. More recently, two studies have simultaneously confirmed that older male patients with comorbidities have a worse prognosis when critically-ill [20, 21]. However, given the extreme heterogeneity in the admission criteria to an ICU and a scattered follow-up in reported studies, it is problematic to make a comparison between these studies and our study's findings: some studies included patients without ARDS, and some included patients who did not need mechanical ventilation.

Overall the present findings are in line with the general feeling that the elderly with comorbidities are the most vulnerable portion of the population when facing Covid-19 infection [22], especially when they are critically ill. 


\section{CONCLUSIONS}

This study reported a mortality rate of $55 \%$ in critically ill COVID-19 patients with ARDS and requiring mechanical ventilation. The study confirms previous findings that older and more comorbid patients represent the population at higher risk of mortality in this setting.

\section{- ACKNOWLEDGEMENTS}

We thank prof. Benoit Boland (Université Catholique de Louvain, Belgium) for his help and support in revising the data and interpreting the results of the statistical analysis.

\section{- CONFLICT OF INTEREST}

None to declare.

\section{REFERENCES}

1. WHO. WHO Timeline - COVID-19. https://www.who.int/newsroom/detail/27-04-2020-who-timeline---covid-19. Published in 2020. Updated April 27th, 2020. Accessed 18/05/2020.

2. Chen N, Zhou M, Dong $X$, et al. Epidemiological and clinical characteristics of 99 cases of 2019 novel coronavirus pneumonia in Wuhan, China: a descriptive study. Lancet. 2020; 395:507-513.

3. Grasselli G, Pesenti A, Cecconi M. Critical Care Utilization for the COVID-19 Outbreak in Lombardy, Italy: Early Experience and Forecast During an Emergency Response. JAMA. 2020.

4. ECDC. Outbreak of acute respiratory syndrome associated with a novel coronavirus, China: first local transmission in the EU/EEA - third update. https://www.ecdc.europa.eu/en/publicationsdata/risk-assessment-outbreak-acute-respiratory-syndromeassociated-novel-1. Published 2020. Accessed 18/05/2020.

5. Sciensano. covid 19-situation epidemiologique. https:// covid-19.sciensano.be/fr/covid-19-situation-epidemiologique. Published 2020. Accessed 01/06/2020.

6. Wunsch H. Mechanical Ventilation in COVID-19: Interpreting the Current Epidemiology. Am J Respir Crit Care Med. 2020; 202:1-4.

7. Quah P, Li A, Phua J. Mortality rates of patients with COVID-19 in the intensive care unit: a systematic review of the emerging literature. Crit Care. 2020; 24:285.

8. WHO. Clinical management of severe acute respiratory infection (SARI) when COVID-19 disease is suspected. https:// www.who.int/docs/default-source/coronaviruse/clinicalmanagement-of-novel-cov.pd. Published 2020. Updated March 13th, 2020. Accessed 01/06/2020.

9. Namendys-Silva SA. Respiratory support for patients with COVID-19 infection. Lancet Respir Med. 2020; 8:e18.

10. Charlson M, Szatrowski TP, Peterson J, Gold J. Validation of a combined comorbidity index. J Clin Epidemiol. 1994; 47:124551.

11. WHO. Clinical management of Covid-19 - Interim Guidance. https://www.who.int/publications/i/item/clinicalmanagement-of-covid-19. Published 2020. Updated May 27th 2020. Accessed 29/06/2020.

12. Yang $X, Y u Y, X u J$, et al. Clinical course and outcomes of critically ill patients with SARS-CoV-2 pneumonia in Wuhan, China: a single-centre, retrospective, observational study. Lancet Respir Med. 2020; 8:475-481.

13. Tu WJ, Cao J, Yu L, Hu X, Liu Q. Clinicolaboratory study of 25 fatal cases of COVID-19 in Wuhan. Intensive Care Med. 2020; 46:1117-1120.

14. Wang Y, Lu X, Li Y, et al. Clinical Course and Outcomes of 344 Intensive Care Patients with COVID-19. Am J Respir Crit Care Med. 2020; 201:1430-1434.

15. Henderson LA, Canna SW, Schulert GS, et al. On the Alert for Cytokine Storm: Immunopathology in COVID-19. Arthritis Rheumatol. 2020; 72:1059-1063.

16. Lodigiani C, Iapichino G, Carenzo L, et al. Venous and arterial thromboembolic complications in COVID-19 patients admitted to an academic hospital in Milan, Italy. Thromb Res. 2020; 191:9-14.

17. Long B, Brady WJ, Koyfman A, Gottlieb M. Cardiovascular complications in COVID-19. Am J Emerg Med. 2020; 38:15041507.

18. Xu J, Yang $X$, Yang $L$, et al. Clinical course and predictors of 60-day mortality in 239 critically ill patients with COVID-19: a multicenter retrospective study from Wuhan, China. Crit Care. 2020; 24:394.

19. Wendel Garcia PD, Fumeaux T, Guerci P, et al. Prognostic factors associated with mortality risk and disease progression in 639 critically ill patients with COVID-19 in Europe: Initial report of the international RISC-19-ICU prospective observational cohort. E Clinical Medicine. 2020.

20. Gupta S, Hayek SS, Wang W, et al. Factors Associated With Death in Critically III Patients With Coronavirus Disease 2019 in the US. JAMA Intern Med. 2020.

21. Grasselli G, Greco M, Zanella A, et al. Risk Factors Associated With Mortality Among Patients With COVID-19 in Intensive Care Units in Lombardy, Italy. JAMA Intern Med. 2020.

22. Nikpouraghdam M, Jalali Farahani A, Alishiri G, et al. Epidemiological characteristics of coronavirus disease 2019 (COVID-19) patients in IRAN: A single centre study. J Clin Virol. 2020; 127:104378. 